\title{
SIMULATION SCHEME FOR QUADRICOPTER CONTROL WITH LABVIEW AND
} X-PLANE

\author{
Davi Ferreira de Castro*, Igor Afonso Acampora Prado*, Pedro Filizola Sousa Maia \\ Gonçalves*, Davi Antônio dos Santos*, Luiz Carlos Sandoval Góes* \\ *Praça Marechal Eduardo Gomes, 50, Vila das Acácias, 12228-900 \\ Instituto Tecnológico de Aeronáutica \\ São José dos Campos, São Paulo, Brasil
}

Emails: davifc@ita.br, igorap@ita.br, pedrofsm@ita.br, davists@ita.br, goes@ita.br

\begin{abstract}
Unmanned Aerial Vehicles (UAV) became interesting for applications where manned operations are considered inefficient and dangerous for humans. This paper is dedicated to one special type of UAV called quadricopter. Lately, this UAV have been in the spotlight, because it has the vertical take-off and landing feature, it can perform hovered flight with changes orientation capability, besides it has high maneuverability and simple mechanic. This paper proposes an virtual simulation environment for validate control techniques applied to quadricopters. This scheme will integrate the virtual environment of X-Plane flight simulator with the mathematical and graphical LabView tooling.
\end{abstract}

Keywords- Quadricopter, Virtual Simulation Environment, Control Techniques, X-Plane, LabView.

Resumo - Veículos aéreos não tripulados (VANT) tornaram-se interessantes para aplicações em operações tripuladas que são considerados ineficientes e perigosas para os seres humanos. Este artigo é dedicado a um tipo especial de VANT chamado de quadricóptero. Ultimamente, este VANT têm sido o centro das atenções, porque tem características de decolagem e pouso verticais, ele pode realizar vôo pairado com capacidade de mudanças de orientação, além disso, tem alta manobrabilidade e simples mecânica. Este trabalho propõe um ambiente virtual de simulação para validar técnicas de controle aplicadas a quadricopters. Este esquema irá integrar o ambiente virtual do simulador de voo X-Plane com o ferramental matemático e gráfico do LabView.

Palavras-chave- Quadricóptero, Ambiente Virtual de Simulação, Técnicas de Controle, X-Plane, LabView.

\section{Introduction}

Unmanned Aerial Vehicles (UAV) became interesting for applications where manned operations are considered inefficient and dangerous for humans. These devices are arousing interest of private companies and the government for search and rescue applications, surveillance of fires in forests, buildings inspection, agricultural services, mapping, naval operations and borders monitoring (Alexis et al., 2011). Other applications are identified such as natural disasters and electrical transmission lines inspection, remote sensor for atmospheric measurements and aerial photography (Tarhan and Altug, 2010).

Among the various UAVs platforms such as: fixed wing ( Teo et al. (2004) and blimp Zufferey et al. (2002) stand out the quadricopters. They are aircrafts heavier than air that have the vertical take-off and landing feature (VTOL). They have four independent and individual rotors. The vehicles movements stem from changes in the propellers rotations. Vertical movement is made by simultaneously increasing (see Fig. 1 (e)) or decreasing (see Fig. 1 (f)) the rotors speeds. Longitudinal motions are achieved by means of changes in the front and rear rotors (see Fig. 1 (a) and (b)), while lateral displacements are performed using the speed of the right and left propellers (see Fig. 1 (c) and (d)). Yaw movement is obtained from unbalances in the counter-torque between each pair of propellers (see Fig. 1 (g) and (h)) (Lopes et al., 2011).

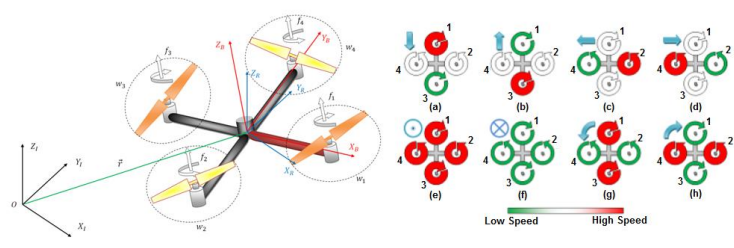

Figure 1: Quadricopter and its movements.

The VTOL systems have specific features that allow varied applications. Therewith, it is possible perform flights of vertical velocity, hovered flight and low-altitude flight. This concept offers a better payload and it is more simple to build and control (Bouabdallah et al., 2004). Its reduced size and high maneuverability allow indoor and outdoor flights (Santana and Braga, 2008). Simulations decrease the development time of new technologies, because it is possible perform tests more quickly and less tiring than practical experiments. Through simulations like Software-In-TheLoop (SIL), it is possible not only validate the system before an experimental test, but also design a compatible hardware and avoid injury and wear for the equipment (Pizeta et al., 2012).

A simulation environment is very important for the avionic system development. This paper proposes an virtual simulation environment SIL for validate control techniques applied to quadricopter. This scheme will integrate the X-Plane 
capability of simulating the aircraft dynamics and providing sensors data as linear and angular velocities, as well as linear and angular positions. The LabView will be utilized for designing control laws and developing graphical interface. The ITA-001 virtual quadricopter model design for Figueiredo and Saotome (2012a) will be utilized in this paper to validate the simulation scheme. This article is organized as follows: Section II describes related concepts. Section III presents system integration. Section IV describes user interface and Section V contains conclusions and suggestions for future work.

\section{Related Concepts}

\section{$2.1 X$ - Plane Flight Simulator}

The demand for flight simulators is increasing due to advance in the processing capability of the computers. The major simulators are: Flight Gear, Microsoft Flight Simulator and X-Plane. This paper chose the X-Plane simulator to implement the simulation environment due to following features: FAA (Federal Aviation Administration) certification, it is possible to simulate real conditions like wind and turbulence and this simulator provide an aircraft construction model called PlaneMaker, based on blade elements where, with the aircraft geometry, engine and propellers parameters, it is possible build the desire aircraft, besides facilitating the simulator data insertion and extraction through User Datagram Protocol (UDP) (Indriyanto and Jenie, 2010).

\subsection{The virtual quadricopter model}

The virtual quadricopter model was designed using the Plane-Maker tool from the X-plane simulator. It was based on the real quadricopter model ITA-001, as shown in Figure 2, which was made from various remote control hobby parts.

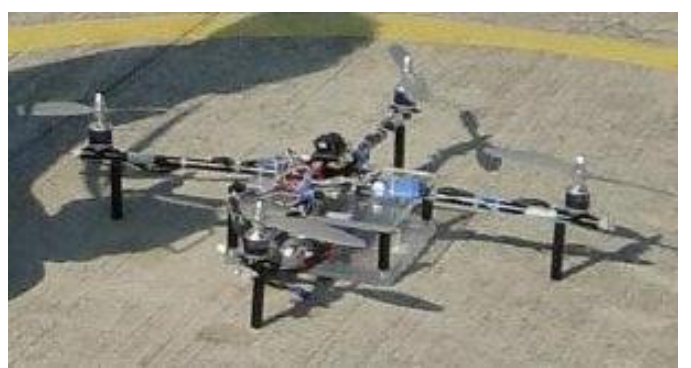

Figure 2: ITA-001.

The ITA-001 quadricopter has four independent fixed-pitch propellers. This feature complicated the quadricopter's modeling, because the Plane-Maker construction module was designed, mainly, for fixed-wing aircrafts modeling, that are the most popular in the X-Plane simulator (Figueiredo and Saotome, 2012a).

The quadricopter model designed in PlaneMaker was validated through comparations with the mathematic model. For a complete development, please refer to (Figueiredo and Saotome, 2012b).

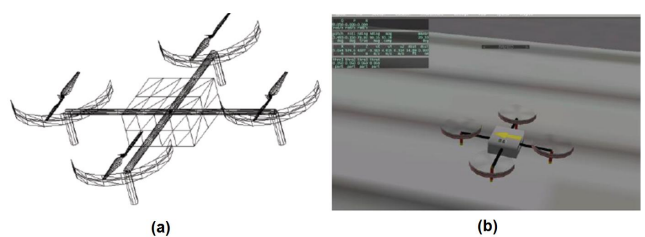

Figure 3: (a) Model quadricopter ITA-001 in Plane-Maker (b) Flight quadricopter ITA-001 in $\mathrm{X}$-Plane.

\subsection{Model Programming - LabView}

LabView is a type of graphical programming language and a tool which can develop a system by doing a design based on data processing flow. This tool provides virtual instruments for implementation of various functions such as signal condition processing to be necessary for data acquisition, A/D conversion, timing operation, storage and communication processing for data analysis, data visualization, and reporting (Shin et al., 2011). Figure 4 shows a sample of data processing procedure using virtual instruments provided by LabView.

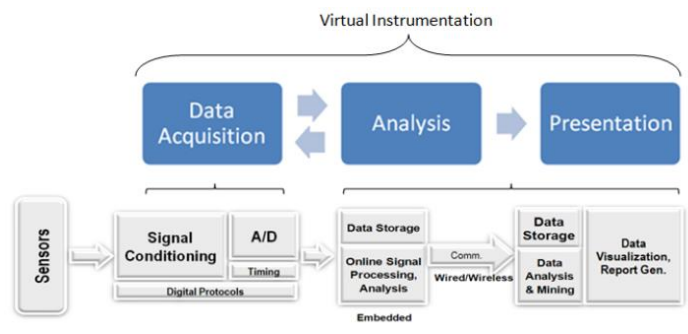

Figure 4: Data processing (Shin et al., 2011).

\section{System Integration}

\subsection{X-Plane/LabView communication}

The X-Plane simulator communicates through User Datagram Protocol (UDP). This protocol is composed just for data and there are not techniques to check if the data was sent correctly, becoming it not reliable. However, the data transmission is very fast, therefore it can be utilized for real-time applications and it is ideal for send and receive X-Plane data. The first step to perform the communication between LabView and 
$\mathrm{X}$-Plane is set the data that will be sent from $\mathrm{X}$ Plane to LabView. This selection is made through "Data Input \& Output" menu (see Figure 5). The data received for LabView will be utilized to calculate the controls needed to stabilize the quadricopter. After selecting all data, it is need setting

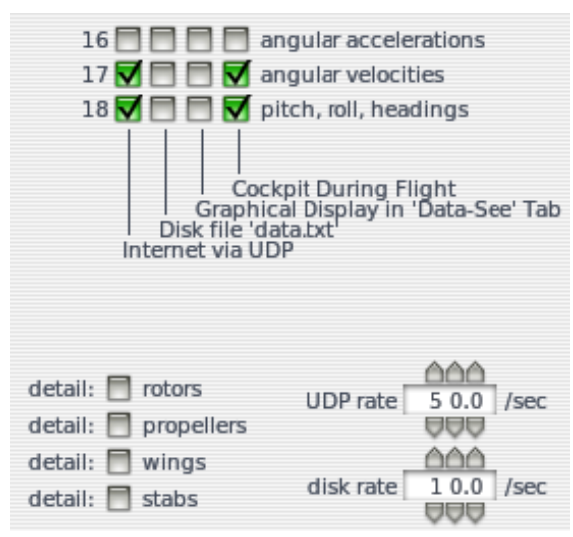

Figure 5: Data selection that will be sent via UDP.

the simulator to send UDP packages. In the XPlane main screen, selecting the "Settings" menu and after "Net Connections", on the Advanced tab, it is inserted the IP (Internet Protocol) address of the machine that will exchange informations with the X-Plane simulator and then it is defined the data sending gate of the simulator. The Figure 6 illustrates these configurations.

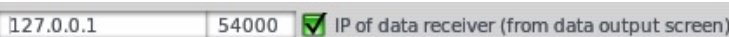

Figure 6: Configurations of IP and sending gate.

\subsection{PID Controller}

It was design a PID (Proportional Integral Derivative) controller to validate the simulation environment. This controller has the following functions: perform the quadricopter altitude control, as well as the control of pitch, roll and yaw angles. The LabView is responsible for calculate the controls that will be applied to the quadricopter and the $\mathrm{X}$-Plane will simulate the quadricopter dynamic. The Figure 7 shows this concept.

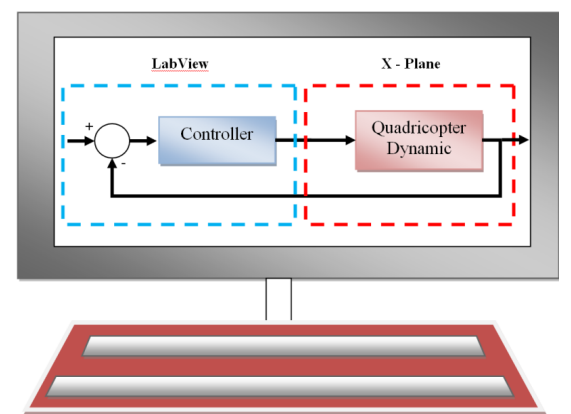

Figure 7: Simulation environment concept.
It was developed four VIs to perform that functions: "PID Altitude", "PID Pitch", "PID Roll" and "PID Yaw". "PID Altitude" is responsible for perform the quadricopter altitude control. Its function is minimizing the error between the altitude reference and the value received from XPlane simulator. "PID Pitch" controls the quadricopter pitch angle, "PID Roll" performs the roll angle control and "PID Yaw" makes the yaw angle control. There is a control loop inside each

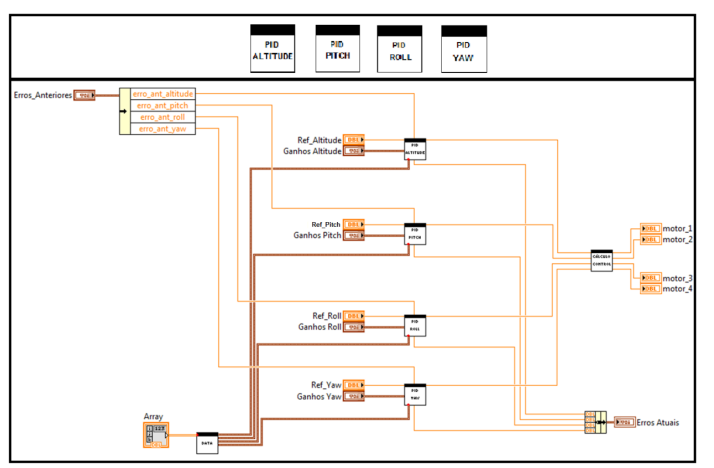

Figure 8: PIDs controls.

PID control with a integral gain performed thorough 4th order Runge-Kutta method, a proportional gain and a derivative gain represented for quadricopter tachometric feedback. This control loop can be seen in the Figure 9.

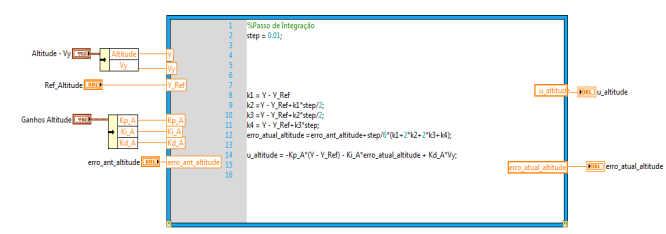

Figure 9: Control Loop PID example.

\section{User Interface}

It was developed an interface for make possible the interaction between the user and the simulation environment. In this interface is possible visualize the following data: engines rotation, artificial horizon, graphics and snapshots data of position, attitude angles, angular and linear velocities. The user has the capability of change the individual gains in each loop control, besides he can set the data receiving gate and the communication IP. The user interface can be seen in the Figure 10 .

\section{Simulation Tests}

In this section, it is proposed simulation tests for one quadricopter with mass $m=1 \mathrm{~kg}$ and the gravitational acceleration is assumed to be $g=9.8 \mathrm{~m} / \mathrm{s}^{2}$. It is worth mentioning that this paper do not focusses on the PID control tuning, it 


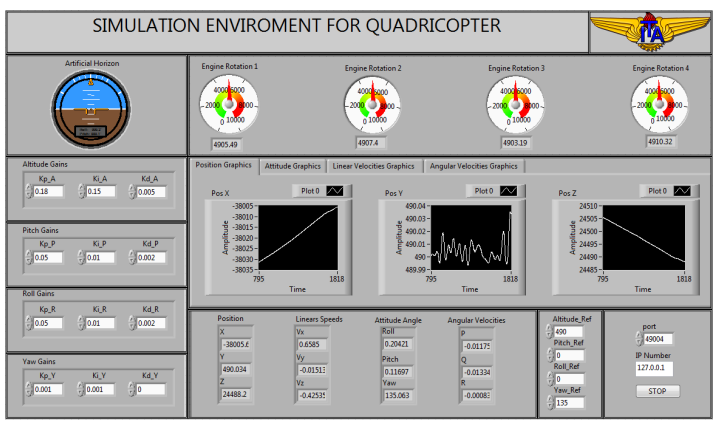

Figure 10: User Interface in Labview.

rather concerns in the development of the virtual simulation environment. In order to validate the virtual simulation environment, one experiment was performed using the PID controller designed. The control architecture is divided in two closed loops, the altitude loop with gains $K_{p}=0.009$, $K_{i}=0.15, K_{d}=0.05$, and the attitude loop with gains $K_{p}=0.01, K_{i}=0.05, K_{d}=0.002$. The PID controller was tuned empirically. The system assumed initial condition $X_{i}=0 \mathrm{~m}, Y_{i}=0$ $m$ and $Z_{i}=0 \mathrm{~m}$, furthermore a step input of $Z_{\text {ref }}=5 \mathrm{~m}$ was applied on the plant in order to evaluate the altitude control. For the attitude, the initial conditions was assumed to be $\operatorname{Roll}_{i}=0^{\circ}$, $\operatorname{Pitch}_{i}=0^{\circ}$ and $\mathrm{Yaw}_{i}=45^{\circ}$, and chosen references were Roll Ref $=0^{\circ}$, Pitch ${ }_{\text {ref }}=0^{\circ}$ and $\mathrm{Yaw}_{\text {ref }}=0^{\circ}$. According to the Figure 11 it is possible to see that the controller was able to track the reference commands.
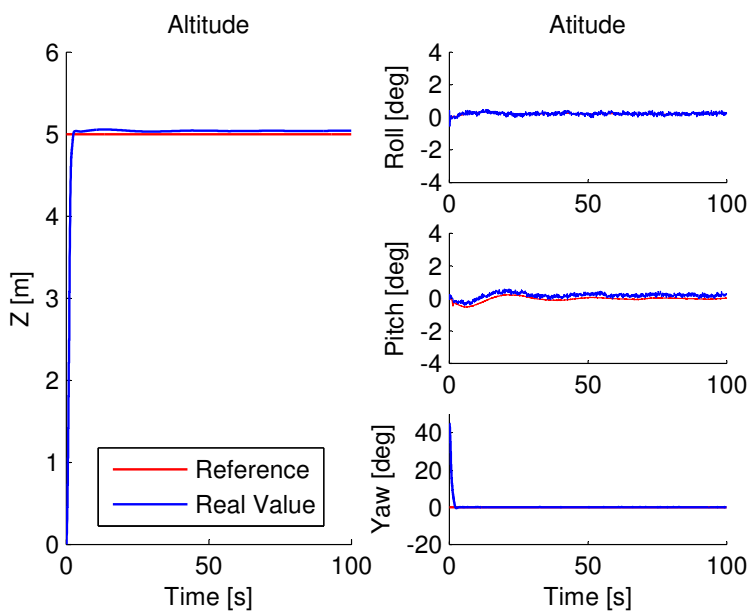

Figure 11: Simulation responses.

The Figure 12 shows the virtual simulation environment in X-Plane

\section{Conclusions}

The simulation environment was presented like an excellent tool for techniques control study applied to quadricopters. As mentioned, the simulation is

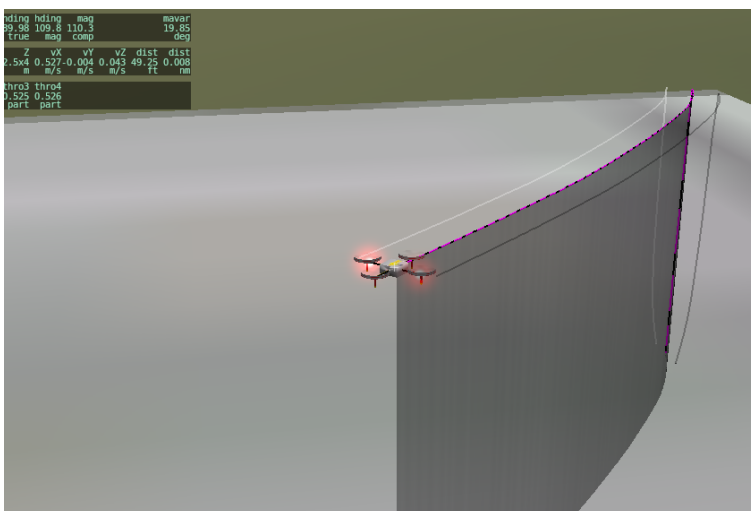

Figure 12: X-Plane Environment.

an efficient manner to validate dynamic model and controllers with saving time when compared to practical experiments. Moreover, it avoids damage and wear to the equipment and presents a quickly visualization of the system behavior. It was presented a complete ambient for control engineers. The platform allowed the behavior analysis of the implemented controller. The simulation environment helped in the UAVs prototyping, once made possible the sensor data inclusion, because the X-Plane simulator provides all required sensor data, as well as it was possible utilized these data to control the quadricopter. The goal of this scheme is assemble all possible sensor informations during the simulations so that adverse situations can be observed and the controller can be effective. For future works, the simulation scheme will be extended for quadricopters swarm and it will be design new controllers with tracking capability and trajectory control, besides change the mechanic behaviors of the quadricopter.

\section{Acknowledgments}

The authors acknowledge the financial support of FAPEAM - Foundation for Research of the Amazon by means of Master Scholarships, as well as the ITA - Aeronautical Institute of Technology for the support needed to carry out this work.

\section{References}

Alexis, K., Papachristos, C., Nikolakopoulos, G. and Tzes, A. (2011). Model predictive quadrotor indoor position control, Proceedings of the 19th Mediterranean Conference on Control and Automation, Corfu, Greece.

Bouabdallah, S., Murrieri, P. and Siegwart, R. (2004). Design and control of an indoor micro quadrotor, IEEE International Conference on Robotics and Automation 5: 43934398 . 
Figueiredo, H. V. and Saotome, O. (2012a). Modelagem e simulação de veículo aéreo não tripulado (vant) do tipo quadricóptero usando o simulador x-plane e simulink, Anais do XIX Congresso Brasileiro de Automática, $C B A$ 2012, Campina Grande, Brazil.

Figueiredo, H. V. and Saotome, O. (2012b). Simulation platform for quadricopter: Using matlab/simulink and x-plane, XIV Simpósio de Aplicações Operacionais em Áreas de Defesa, SIGE 2012, São José dos Campos, Brazil.

Indriyanto, T. and Jenie, Y. I. (2010). Modeling and simulation of a ducted fan unmanned aerial vehicle ( uav ) using x-plane simulation software, Regional Conference on Mechanical and Aerospace Technology, Bali.

Lopes, R. V., Santana, P. H. R. Q. A., Borges, G. A. and Ishihara, J. Y. (2011). Model predictive controle applied to tracking and attitude stabilization of a vtol quadrotor aircraft, Proceedings of the 21st International Congress of Mechanical Engineering COBEM, Natal, Brazil.

Pizeta, I. H. B., Brandao, A. S., Filho, M. S. and Carelli, R. (2012). Uma plataforma hardware in loop para vants de asas rotativas, Anais do XIX Congresso Brasileiro de Automatica, $C B A$ 2012.

Santana, P. H. R. Q. A. and Braga, M. A. (2008). Concepção de um veículo aéreo nãotripulado do tipo quadrirrotor, Trabalho de

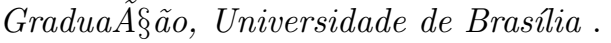

Shin, Hyeon-gab, chul Park, M., soo Jun, J., ho Moon, Y. and Ha, S.-W. (2011). Implementation of an integrated test bed for avionics system development, Software Engineering, Business Continuity, and Education, Jeju Island, Korea.

Tarhan, M. and Altug, E. (2010). Model predictive quadrotor indoor position control, Journal of Intelligent and Robotic Systems 62: 587-607. DOI: 10.1007/s10846-010-9459-y

Teo, R., Jang, J. S. and Tomlin, C. J. (2004). Model predictive quadrotor indoor position control, Proc 43rd IEEE Conf. Decision Control, Paradise Island, Bahamas 4: 4268-4273.

Zufferey, J. C., Floreano, D., van Leeuwen, M. and Merenda, T. (2002). Evolving vision-based flying robots, Proceedings of the 2nd International Workshop on Biologically Motivated Computer Vision-Springer-Verlag Berlin Heidelberg pp. 592-600. 